\title{
Color preferences in participants with high or low hypnotic susceptibility
}

This article was published in the following Dove Press journal:

Neuropsychiatric Disease and Treatment

\author{
Enyan $\mathrm{Yu}^{1,2}$ \\ Junpeng Zhu ${ }^{1,2}$ \\ Yunfei Tan ${ }^{1,2}$ \\ Zhengluan Liao ${ }^{1,2}$ \\ Yaju Qiu',2 \\ Bingren Zhang ${ }^{3}$ \\ Chu Wang ${ }^{3}$ \\ Wei Wang ${ }^{3}$ \\ 'Department of Psychiatry, Zhejiang \\ Provincial People's Hospital, \\ Hangzhou, People's Republic of China; \\ ${ }^{2}$ Department of Psychiatry, \\ People's Hospital of Hangzhou \\ Medical College, Hangzhou, \\ People's Republic of China; \\ ${ }^{3}$ Department of Clinical Psychology \\ and Psychiatry/School of Public \\ health, Zhejiang University \\ College of Medicine, Hangzhou, \\ People's Republic of China
}

Correspondence: Wei Wang

Department of Clinical Psychology

and Psychiatry/School Public Health,

Zhejiang University College of Medicine,

Yuhangtang Road 866, Hangzhou,

Zhejiang 31 10058, People's Republic

of China

Tel +86 57l 88208188

Email drwangwei@zju.edu.cn;

wangmufan@msn.com
Purpose: Color preferences vary among normal individuals and psychiatric patients, and this might be related to their different levels of hypnotic susceptibility. We hypothesized that individuals with higher hypnotic susceptibility prefer more arousing colors such as red.

Patients and methods: Out of 440 participants, we selected 70 with higher (HIGH) and 66 with lower (LOW) hypnotic susceptibilities, and asked them to undergo the Stanford Hypnotic Susceptibility Scale: Form C (SHSSC) test, then to order their preferences of 11 colors.

Results: The HIGH group preferred red more and scored higher on the total SHSSC. The preference order of black was negatively predicted by the SHSSC Taste hallucination but positively by Arm rigidity, and the preference of yellow was positively predicted by Posthypnotic amnesia and Taste hallucination in the HIGH group.

Conclusion: The red preference and the SHSSC associations with black and yellow preferences in participants with high hypnotic susceptibility help to clarify the individual difference of color preference and provide research hints for behavioral studies in normal individuals and psychiatric patients.

Keywords: color perception, healthy people, the Stanford Hypnotic Susceptibility Scale: Form C (SHSSC)

\section{Introduction}

Color is a fundamental aspect of human perceptual experience of external world. ${ }^{1}$ Color preference, as a part of many consumer and aesthetic choices in daily lives, is crucial for understanding people's behavior; for instance, it has an effect on judgments and decisions of an individual, such as which clothes to wear or which product to buy. ${ }^{2,3}$ Investigators are trying to figure out colors people preferring and the reasons for doing so. For instance, some scholars have shown that the average color preference followed a relatively smooth and curvilinear function, with a peak at blue and a trough around yellow or yellow-green..$^{3-7}$ Other scholars have found a cross-cultural pattern of sex difference on the color preference, eg, men preferred green and women preferred red. ${ }^{4,8}$

There are also some models which might explain the pattern of color preference. One model is the ecological valence theory, ${ }^{5}$ which describes people like/dislike colors to a degree that is associated with the liked/disliked objects in these respective colors. The association might have an ecological and adaptive function through the individual learning mechanism, which is the repeated pairing of color and particular concepts, messages, and experiences over time. ${ }^{9}$ However, the ecological valence theory is challenged by the results that the color preference varies between different cultures. ${ }^{10}$ Some scholars have described different symbolic and conceptual 
associations of colors in several cultures. ${ }^{7,11}$ Indeed, when people frequently encounter situations where different colors are accompanied by particular objects or experiences, they would form specific associations to those colors. Therefore, the culture-specific personal experiences would influence his/her color preference.

Further, other factors, such as personality traits, are related to the color preference. Fetterman et $\mathrm{al}^{12}$ found that people preferring red over blue were more interpersonally hostile, which might be due to that red was linked to anger metaphorically and physiologically. Another study has shown that the connections between color preference and personality traits fit into a social structural layout, for example, males preferred stimulating colors such as yellow in order to elevate their sociability levels. ${ }^{13}$ On the other hand, mental disorders or psychopathological alterations also influence the color preference. ${ }^{14}$ For instance, one study has shown psychotic patients preferred green, ${ }^{15}$ another has shown patients with mild depression preferred black and brown. ${ }^{16}$

Furthermore, the hypnotic susceptibility is a stable ability of responsiveness to suggestions for changes in subjective experience, and for alterations in perception, sensation, emotion, thought, or behavior. ${ }^{17,18}$ High hypnotizables were reported to have increased accessibility to affective events and exaggerated emotional feelings. ${ }^{19}$ Considering color preference is determined by the average affective response to everything the individual associates with the color, ${ }^{5}$ it might be influenced by individual hypnotizability. Similar to personality function, which varies from normal to diseased conditions, the hypnotic susceptibility also displays a continuum from low to high states; therefore, different states of hypnosis might be associated with different patterns of color preference. Previous studies have demonstrated that the hypnotic susceptibility is correlated with normal personality traits such as the openness to experience. ${ }^{20,21}$ Interestingly, red as an arousing color ${ }^{22,23}$ is correlated mostly with the trait of thrill and adventure seeking. ${ }^{24}$ Moreover, higher hypnotic susceptibility has been reported in patients suffering from posttraumatic stress disorder, ${ }^{25}$ and lower hypnotic susceptibility has been reported in schizophrenia patients, ${ }^{26}$ who preferred black, red, and white ${ }^{27}$ or brown. ${ }^{28}$ These outcomes again imply that color preferences are different in individuals with different hypnotic susceptibilities.

In the current study, we have hypothesized that individuals with higher hypnotic susceptibility prefer more arousing colors such as red than those with lower one do. We therefore have invited participants with either high or low hypnotic susceptibility to undergo hypnosis, and later to undergo the color preference test. There are many scales assessing hypnotic susceptibility, and in the current study we chose the Stanford Hypnotic Susceptibility Scale: Form C (SHSSC), ${ }^{29}$ which is one of the most widely used measures of this kind. ${ }^{30}$ Consistent with previous studies, ${ }^{13,31}$ we chose eleven basic colors, ie, white, gray, black, red, green, blue, yellow, orange, pink, brown, and purple, to measure the color preference of our participants.

\section{Patients and methods \\ Participants}

The first three SHSSC items, ie, the Hand lowering, Moving hands apart, and Mosquito hallucination (see the "Hypnosis test" section), were used for screening participants due to the ascending order of item difficulty and the good predictions of the first three items for the full SHSSC test..$^{32}$ Those who either had passed two items (high susceptibility) or had failed to pass any item (low susceptibility) were enrolled into the current study, as per the procedure in a previous report. ${ }^{21}$ Altogether 440 participants from university or community were invited, only 136 participants succeeded to be enrolled: 70 were classified into the high hypnotic susceptibility group (HIGH; 27 men, mean age: 21.04 years $\pm 1.65 \mathrm{SD}$, ranged 18-25 years); and 66 into the low hypnotic susceptibility group (LOW; 28 men, mean age: $21.06 \pm 1.62$, ranged $18-26)$. There was no age $(t=0.06, d f=134, p=0.95)$ or gender $\left(\chi^{2}=0.21, p=0.65\right)$ difference between groups. Participation was restricted to individuals with normal color vision as verified on the color vision test. A semi-structured interview was performed for each participant in order to ascertain that they were not suffering or had not suffered from any psychiatric problems, including personality disorder. All participants were naive to the study design and had normal or correctedto-normal visual acuity. The study has been approved by the Ethics Committee of Zhejiang University College of Medicine, and all participants gave their written informed consents to participate in the study.

\section{Procedures}

\section{Hypnosis test}

The Chinese version of the SHSSC used previously was applied in the current study. ${ }^{21,33}$ The SHSSC consists of 12 dichotomously scored items including direct ideomotor (eg, arm heaviness), challenge motor (eg, arm immobilization), and cognitive-perceptual (eg, auditory hallucination) suggestions, with scores ranging from 0 to 12 . The internal $\alpha$ (reliability) of SHSSC was 0.85 in the current study. 
If a participant got one point on an item, we referred to it as "s/he has passed the item," otherwise as "s/he has failed to pass the item." We defined "passing rate" of each item as the percentage of participants who had passed that item.

\section{Color preference test}

The participant was positioned about $50 \mathrm{~cm}$ from the monitor in a dark room with the illumination consisting of the direct, diffused light from $40 \mathrm{~W}$ fluorescent lamps. The colors were presented on a computer monitor $(1,280 \times 800$ pixels, vertical refresh rate, $75 \mathrm{~Hz}$ ). The background color of the screen was kept constantly in gray (Red: 153; Green: 153; Blue: 153; Hue: 0; Saturation: 0; Brightness: 60). The color was formulated using the Adobe Photoshop CS3 extended edition software (Adobe Systems, San Jose, CA, USA). At the beginning of the experiment, participants were given 3 minutes to adapt to the background color, and 3 minutes later for the 11 target colors presented against the gray background. All target colors (ie, white, gray, black, red, green, blue, yellow, orange, pink, brown, and purple) were displayed as rectangular patches $\left(2.5 \times 2.5 \mathrm{~cm}^{2}\right.$ each $)$, and the chromatic scheme values of Red-Green-Blue and of Hue-Saturation-Brightness for these colors were in accordance with the former study. ${ }^{13}$ Each color was presented in one patch for 1 minute in the middle of the screen against a gray background, and the patch presentations were randomized among participants. Then the participants were asked to write down their preference orders of these colors, from 11 to 1 , beneath the name of these colors on a piece of paper, with the bigger number representing the more preferred one. There was no time limitation on the response, but participants were asked to select as quickly as possible without cogitation and particularly without reference to any other color-vectors outside the monitor.

\section{Statistical analyses}

The $\chi^{2}$ test was used to evaluate the differences of SHSSC item passing rates in the LOW and HIGH groups. The differences of the color preference rank orders between the two groups were tested with Mann-Whitney $U$ test. The relationships between scores of SHSSC and the preference orders were assessed by multiple linear regression analysis (stepwise method) in a given group. The $p$-value $<0.05$ was considered to be significant.

\section{Results}

The Mann-Whitney $U$ test detected significant group differences with regard to the color red $(U=1,572.00, p=0.001)$, but showed no difference in other colors (Figure 1). The HIGH group preferred Red (7.79 \pm 3.14$)$ more than the LOW group (6.08 \pm 3.06$)$ did. Not surprisingly, the total SHSSC score in the HIGH group $(8.77 \pm 0.84)$ was higher $(t=42.25$, $d f=134, p<0.05)$ than that in the LOW group $(2.09 \pm 1.00)$. Comparing the passing rates of SHSSC items, the HIGH group passed all significantly more often than the LOW group did (Table 1).

In HIGH group, the preference order of black was negatively predicted by the SHSSC Taste hallucination (adjusted $R^{2}=0.12, \beta=-0.31$, standard error $(\mathrm{SE})=1.06$, $p=0.008,95 \%$ confidence interval $(\mathrm{CI})=[-5.02$ to -0.80$])$ and positively by Arm rigidity $(\beta=0.25, \mathrm{SE}=1.20, p=0.033$, $95 \% \mathrm{CI}=[0.21-5.01])$. The preference order of yellow was positively predicted by Posthypnotic amnesia (adjusted $\left.R^{2}=0.11, \beta=0.30, \mathrm{SE}=0.66, p=0.012,95 \% \mathrm{CI}=[0.38-3.00]\right)$

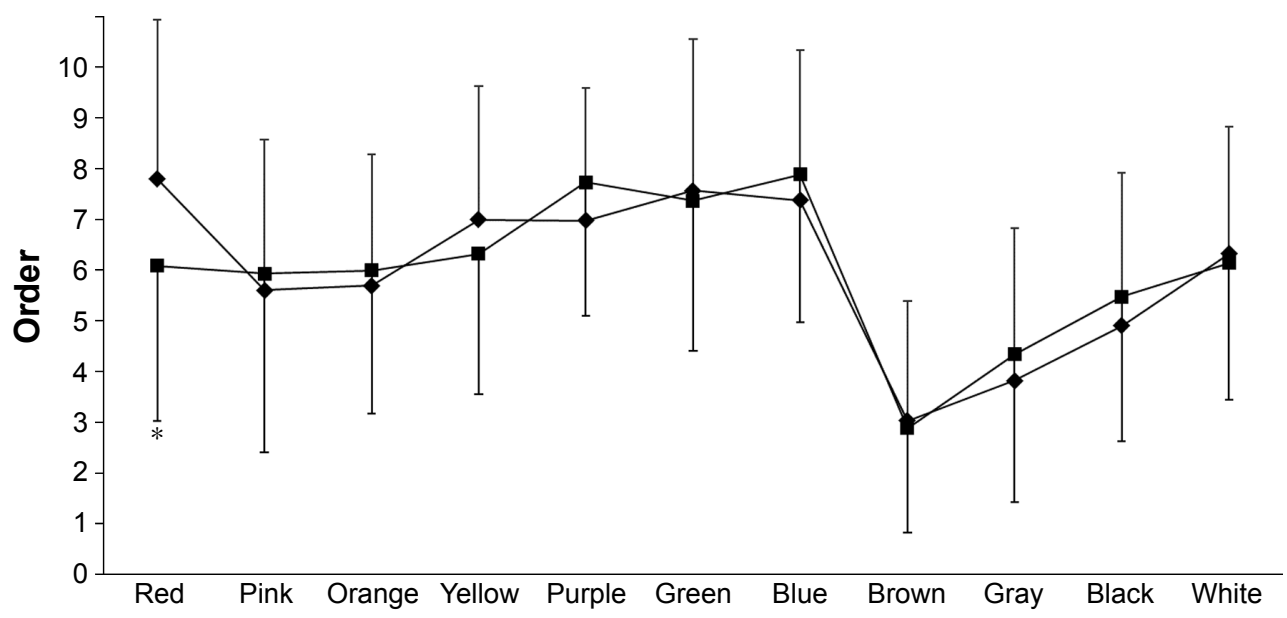

Figure I Color preference orders in the HIGH (rhombus, $n=70$ ) and LOW (square, n=66) groups.

Notes: ${ }^{*} p<0.05$ vs HIGH group. HIGH group consisted of participants with higher hypnotic susceptibilities; LOW group consisted of participants with lower hypnotic susceptibilities. 
Table I Distribution of participants who passed the SHSSC test in the two groups of participants

\begin{tabular}{llll}
\hline SHSSC item & \multicolumn{2}{l}{ Number, rate, \% } & $\chi^{2}$ \\
\cline { 2 - 3 } & $\begin{array}{l}\text { HIGH } \\
(\mathbf{n}=\mathbf{7 0})\end{array}$ & $\begin{array}{l}\text { LOW } \\
(\mathbf{n}=\mathbf{6 6})\end{array}$ & \\
\hline Hand lowering & $61,87.1$ & $22,33.3$ & 41.4 \\
Moving hands apart & $65,92.9$ & $17,25.8$ & 63.9 \\
Mosquito hallucination & $64,91.4$ & $14,21.2$ & 68.5 \\
Taste hallucination & $62,88.6$ & $13,19.7$ & 65.1 \\
Arm rigidity & $64,91.4$ & $15,22.7$ & 65.9 \\
Dream & $42,60.0$ & $3,4.5$ & 47.2 \\
Age regression & $67,95.7$ & $36,54.5$ & 31.3 \\
Arm immobilization & $63,90.0$ & $4,6.1$ & 95.8 \\
Anosmia to ammonia & $37,52.9$ & $5,7.6$ & 32.6 \\
Hallucinated voice & $21,30.0$ & $3,4.5$ & 15.1 \\
Negative visual hallucination & $47,67.1$ & $5,7.6$ & 51.0 \\
Posthypnotic amnesia & $21,30.0$ & $1,1.5$ & 20.3 \\
\hline Notes: Al &
\end{tabular}

Notes: All $p$-values $<0.01$ vs LOW group. HIGH group consisted of participants with higher hypnotic susceptibilities; LOW group consisted of participants with lower hypnotic susceptibilities.

Abbreviation: SHSSC, Stanford Hypnotic Susceptibility Scale: Form C.

and Taste hallucination $(\beta=0.27, \mathrm{SE}=0.94, p=0.023$, $95 \% \mathrm{CI}=[0.32-4.08])$. Some colors could be predicted by the SHSSC items, but these associations were not reported here due to their lower determination coefficients (adjusted $R^{2}<0.10$, Table 2).

\section{Discussion}

Compared to the LOW group, the HIGH group preferred red more in our study, which confirmed our hypothesis. The HIGH group also scored higher on the total SHSSC as we expected. However, the weak associations between the preference of red and the SHSSC items offered little explanation for the red preference in the HIGH group. Nonetheless, the preference order of black was negatively predicted by Taste hallucination but positively by Arm rigidity, while the preference order of yellow was positively predicted by Posthypnotic amnesia and Taste hallucination in the HIGH group.
Red is the color of blood and fire, and is considered a signal conveying danger and provoking avoidance, ${ }^{34}$ which seems to be of particular significance for survival. ${ }^{23,35}$ In competitive contexts, red functions as a dominance cue and might enhance performance accordingly. ${ }^{22}$ In other circumstances, red is considered as healthy, ${ }^{36}$ attractive, and sexually desirable. ${ }^{9}$ In these normal situations, red color has a high arousal level which is connected with adventure seeking ${ }^{23,24}$ this might help to explain our finding of the higher red preference in the HIGH group..$^{21}$ In clinics, the high hypnotizability was correlated with posttraumatic stress disorder ${ }^{37}$ and dissociative disorder, ${ }^{38}$ and also children ${ }^{27}$ and veterans $^{39}$ with posttraumatic stress disorder consistently preferred the color red.

Some previous studies support the associations between the preference of black and Taste hallucination (-) and Arm rigidity found in the HIGH group. Black is associated with negative concepts such as evil and death, and it was found to have association with aggression; ${ }^{40}$ while Taste hallucination involves experience of sweet and sour tastes and either one strong or one with movements, which demands much devotion and fantasy. ${ }^{29,33}$ Although we failed to record the aggression trait in our participants, the preference of black might reflect that these participants did not comply with the induction of cognitive alteration. Therefore, the preference order of black was negatively predicted by Taste hallucination in the HIGH group. Arm rigidity is a motor challenge item, which requires the participant to imagine her/his arm to be stiff and rigid and thus they cannot overbend it. ${ }^{29}$ It is an easier one to pass since the individual needs to do nothing during the hypnosis induction, ${ }^{41}$ which might particularly be the case in our participants with high hypnotic susceptibility.

In our HIGH group, the associations between the preference of yellow and Posthypnotic amnesia and Taste hallucination were also supported by previous results.

Table 2 Stepwise multiple regression among color preferences and the SHSSC items in the HIGH ( $n=70)$ and LOW ( $n=66)$ groups

\begin{tabular}{|c|c|c|c|c|c|c|c|}
\hline Group & $\begin{array}{l}\text { Color } \\
\text { preference }\end{array}$ & SHSSC predictor & $\begin{array}{l}\text { Adjusted } \\
R^{2}\end{array}$ & $\beta$ & SE & $p$-value & $95 \% \mathrm{Cl}$ \\
\hline \multirow[t]{2}{*}{$\mathrm{HIGH}$} & Black & Taste hallucination & 0.12 & -0.31 & 1.06 & 0.008 & -5.02 to -0.80 \\
\hline & & Arm rigidity & & 0.25 & 1.20 & 0.033 & $0.2 I-5.0 I$ \\
\hline $\mathrm{HIGH}$ & White & Arm rigidity & 0.05 & 0.24 & 1.05 & 0.042 & $0.08-4.26$ \\
\hline $\mathrm{HIGH}$ & Brown & Anosmia to ammonia & 0.07 & 0.29 & 0.54 & 0.014 & $0.29-2.46$ \\
\hline $\mathrm{HIGH}$ & Pink & Posthypnotic amnesia & 0.04 & -0.24 & 0.76 & 0.046 & -3.05 to -0.03 \\
\hline \multirow[t]{2}{*}{$\mathrm{HIGH}$} & Yellow & Posthypnotic amnesia & 0.11 & 0.30 & 0.66 & 0.012 & $0.38-3.00$ \\
\hline & & Taste hallucination & & 0.27 & 0.94 & 0.023 & $0.32-4.08$ \\
\hline $\mathrm{HIGH}$ & Purple & Negative visual hallucination & 0.07 & -0.29 & 0.64 & 0.015 & -2.87 to -0.32 \\
\hline LOW & Blue & Moving hands apart & 0.06 & 0.28 & 0.79 & 0.025 & $0.24-3.41$ \\
\hline
\end{tabular}

Notes: HIGH group consisted of participants with higher hypnotic susceptibilities; LOW group consisted of participants with lower hypnotic susceptibilities.

Abbreviations: $\mathrm{Cl}$, confidence interval; SE, standard error of the mean; SHSSC, Stanford Hypnotic Susceptibility Scale: Form C. 
Posthypnotic amnesia examines if one can recall three or fewer items from before the instruction of memory-retrieving was given after the hypnosis test. ${ }^{29}$ It was positively associated with extroversion trait and negatively with openness to experience. ${ }^{21}$ Extroverts preferred yellow since they considered the color exciting. ${ }^{42}$ Similarly, sociability trait was negatively associated with the preference of yellow in males. ${ }^{13}$ The Posthypnotic amnesia score was found positively associated with scores on schizoid personality disorder, ${ }^{33}$ and individuals with this disorder are characterized by unsocial and introverted features. ${ }^{43}$ Moreover, during an electroencephalogram test, the extraverts followed the test instructor with less attention; ${ }^{44}$ the ignoring of the instruction details might facilitate the participants to pass the Taste hallucination test in our HIGH group.

\section{Conclusion}

However, there are several design limitations of the current study which should be noted. First, we conducted a rankordering task to evaluate color preferences, and this might suffer from the ceiling effect since most favorite colors, such as blue and green, are highly preferred ones. Second, we did not record either normal or disordered personalities of our participants, and so whether these traits influence our current results remains unclear. Third, the majority of our participants were university students, and so the current study results cannot be directly generalized to other age ranges. Fourth, all participants were Chinese and the sample sizes for each group were small; thus, whether our findings are unique to Chinese culture remains unanswered. Nevertheless, we have demonstrated that individuals with higher hypnotic susceptibility preferred red more and some of their hypnotic aspects contributed to their preferences of black and yellow. Our study might open new ways to evaluate the characteristics of individuals who are hypnotized, to identify other elements that influence the color preference, and to provide hints for using color arrangements to help the application of cognitive-behavioral therapy to different psychiatric patients with different levels of hypnotic susceptibility.

\section{Acknowledgment}

Dr Enyan Yu was supported by a grant from the Natural Science Foundation of China (No 81771158), and Dr Wei Wang was supported by a grant from the Natural Science Foundation of China (No 91132715).

\section{Author contributions}

WW conceived the study, EY, JZ, YT, ZL, YQ, BZ, and CW contributed to the study design and collected the data, EY,
JZ, and WW analyzed the data, EY, JZ, and WW drafted the paper. EY and JZ contributed equally to the study. All authors read and approved the final manuscript. All authors contributed toward data analysis, drafting and critically revising the paper and agree to be accountable for all aspects of the work.

\section{Disclosure}

Regarding research work described in the paper, each one of our coauthors declares that there is no conflict of interest, has conformed to the Helsinki Declaration concerning human rights and informed consent, and has followed correct procedures concerning treatment of humans in research. The authors report no conflicts of interest in this work.

\section{References}

1. Schulz MF, Sanocki T. Time course of perceptual grouping by color. Psychol Sci. 2003;14(1):26-30.

2. Sable P, Akcay O. Response to color: literature review with crosscultural marketing perspective. Int Bull Bus Adm. 2011;11:34-41.

3. Palmer SE, Schloss KB, Sammartino J. Visual aesthetics and human preference. Ann Rev Psychol. 2013;64:77-107.

4. Hurlbert AC, Ling Y. Biological components of sex differences in color preference. Curr Biol. 2007;17(16):R623-R625.

5. Palmer SE, Schloss KB. An ecological valence theory of human color preference. Proc Natl Acad Sci US A. 2010;107(19):8877-8882.

6. Taylor C, Franklin A. The relationship between color-object associations and color preference: further investigation of ecological valence theory. Psychon Bull Rev. 2012;19(2):190-197.

7. Schloss KB, Hawthorne-Madell D, Palmer SE. Ecological influences on individual differences in color preference. Atten Percept Psychophys. 2015;77(8):2803-2816.

8. Sorokowski P, Sorokowski A, Witzel C. Sex differences in color preferences transcend extreme differences in culture and ecology. Psychon Bull Rev. 2014;21(5):1195-1201.

9. Elliot AJ, Maier MA. Color psychology: effects of perceiving color on psychological functioning in humans. Annu Rev Psychol. 2014;65: 95-120.

10. Taylor C, Clifford A, Franklin A. Color preferences are not universal. J Exp Psychol Gen. 2012;142(4):1015-1027.

11. Yokosawa K, Schloss KB, Asano M, Palmer SE. Ecological effects in cross-cultural differences between U.S. and Japanese color preferences. Cogn Sci. 2016;40(7):1590-1616.

12. Fetterman AK, Liu T, Robinson MD. Extending color psychology to the personality realm: interpersonal hostility varies by red preferences and perceptual biases. J Pers. 2015;83(1):106-116.

13. He W, Zhang Y, Zhu J, et al. Could sex difference in color preference and its personality correlates fit into social theories? Let Chinese university students tell you. Pres Indiv Differ. 2011;51:154-159.

14. French AP. Color rejection revisited. Am J Forensic Psychol. 1996; 14:73-74.

15. Kuloğlu M, Atmaca M, Tezcan AE, Unal A, Gecici O. Color and number preferences of patients with psychiatric disorders in eastern Turkey. Percept Mot Skills. 2002;94(1):207-213.

16. Nolan RF, Dai Y, Stanley PD. An investigation of the relationship between color choice and depression measured by the Beck Depression Inventory. Percept Mot Skills. 1995;81(3 Pt 2):1195-1200.

17. Piccione C, Hilgard ER, Zimbardo PG. On the degree of stability of measured hypnotizability over a 25-year period. J Pers Soc Psychol. 1989; 56(2):289-295. 
18. Green JP, Barabasz AF, Barrett D, Montgomery GH. Forging ahead: the 2003 APA Division 30 definition of hypnosis. Int J Clin Exp Hypn. 2005;53(3):259-264.

19. De Pascalis V. Psychophysiological correlates of hypnosis and hypnotic susceptibility. Int J Clin Exp Hypn. 1999;47(2):117-143.

20. Nordenstrom BK, Council JR, Meier BP. The "big five" and hypnotic susceptibility. Int J Clin Exp Hypn. 2002;50(3):276-281.

21. Zhang Y, Wang Y, Shen C, et al. Relationship between hypnosis and personality trait in participants with high or low hypnotic susceptibility. Neuropsychiatr Dis Treat. 2017;13:1007-1012.

22. Hill RA, Barton RA. Psychology: red enhances human performance in contests. Nature. 2005;435(7040):293.

23. Kuhbandner C, Spitzer B, Lichtenfeld S, Pekrun R. Differential binding of colors to objects in memory: red and yellow stick better than blue and green. Front Psychol. 2015;6:231.

24. Rosenbloom T. Color preferences of high and low sensation seekers. Creativity Res J. 2006;18(2):229-235.

25. Spiegel D, Hunt T, Dondershine HE. Dissociation and hypnotizability in posttraumatic stress disorder. Am J Psychiatry. 1988;145(3): 301-305.

26. Frischholz EJ, Lipman LS, Braun BG, Sachs RG. Psychopathology, hypnotizability, and dissociation. Am J Psychiatry. 1992;149(11): 1521-1525.

27. Gregoryan VS, Azaryan A, DeMaria MB, MsDonald LD. Colors of disaster: the psychology of the "Black Sun." Art Psychother. 1996; 23(1):1-14.

28. Tao B, Xu S, Pan X, Gao Q, Wang W. Personality trait correlates of color preference in schizophrenia. Transl Neurosci. 2015;6(1):174-178.

29. Weitzenhoffer AM, Hilgard ER. Stanford Hypnotic Susceptibility Scale: Form C. Palo Alto, CA: Consulting Psychologists Press; 1962.

30. Champigny CM, Raz A. Transcultural factors in hypnotizability scales: limits and prospects. Am J Clin Hypn. 2015;58(2):171-194.

31. Hardin CL. Basic color terms and basic color categories. In: Backhaus WGK, Reinhold K, Werner JS, editors. Color Vision: Perspectives from Different Disciplines. Berlin: de Gruyter; 1988:207-217.

32. Hilgard ER. The Stanford hypnotic susceptibility scales as related to other measures of hypnotic responsiveness. Am J Clin Hypn. 1978; 21(2-3):68-83.
33. Wang F, Chen WZ, Huang J, et al. Preliminary study of relationships between hypnotic susceptibility and personality disorder functioning styles in healthy volunteers and personality disorder patients. BMC Psychiatry. 2011;11(1):121.

34. Elliot AJ, Maier MA, Binser MJ, Friedman R, Pekrun R. The effect of red on avoidance behavior in achievement contexts. Pers Soc Psychol Bull. 2009;35(3):365-375.

35. Moller AC, Elliot AJ, Maier MA. Basic hue-meaning associations. Emotion. 2009;9(6):898-902.

36. Stephen ID, Coetzee V, Law Smith M, Perrett DI. Skin blood perfusion and oxygenation colour affect perceived human health. PLoS One. 2009;4(4):e5083.

37. Stutman RK, Bliss EL. Posttraumatic stress disorder, hypnotizability, and imagery. Am J Psychiatry. 1985;142(6):741-743.

38. Bliss EL. Multiple personalities, related disorders, and hypnosis. Am J Clin Hypn. 1983;26(2):114-123.

39. Mandic-Gajic G, Spiric Z. Posttraumatic stress disorder and art group therapy: self-expression of traumatic inner world of war veterans. Vojnosanit Pregl. 2016;73(8):757-763.

40. Webster DG, Urland GR, Correll J. Can uniform color cause aggression? Quasi-experimental evidence from professional ice hockey. Soc Psychol Personal Sci. 2012;3(3):274-281.

41. Galea V, Woody EZ, Szechtman H, Pierrynowski MR. Motion in response to the hypnotic suggestion of arm rigidity: a window on underlying mechanisms. Int J Clin Exp Hypn. 2010;58(3):251-268.

42. Eysenck HJ. Aesthetic preferences and individual differences. In: O'Hare D, editor. Psychology and the Arts. Brighton, Sussex: Harvester Press; 1981:76-101.

43. American Psychiatric Association. Diagnostic and Statistical Manual of Mental Disorders. 5th ed. Washington, DC: American Psychiatric Association; 2013.

44. De Pascalis V, Imperiali MG. Personality, hypnotic susceptibility and EE responses: preliminary study. Percept Mot Skills. 1984;59(2): $371-378$.
Neuropsychiatric Disease and Treatment

\section{Publish your work in this journal}

Neuropsychiatric Disease and Treatment is an international, peerreviewed journal of clinical therapeutics and pharmacology focusing on concise rapid reporting of clinical or pre-clinical studies on a range of neuropsychiatric and neurological disorders. This journal is indexed on PubMed Central, the 'PsycINFO' database and CAS,

\section{Dovepress}

and is the official journal of The International Neuropsychiatric Association (INA). The manuscript management system is completely online and includes a very quick and fair peer-review system, which is all easy to use. Visit http://www.dovepress.com/testimonials.php to read real quotes from published authors. 